Check for updates

Cite this: RSC Adv., 2020, 10, 37923

\title{
Improving the performance of proton exchange membrane water electrolyzers with low Ir-loaded anodes by adding PEDOT:PSS as electrically conductive binder $\dagger$
}

\author{
Edgar Cruz Ortiz, ${ }^{a}$ Friedemann Hegge, ${ }^{a}$ Matthias Breitwieser ${ }^{\mathrm{ab}}$ \\ and Severin Vierrath (D) *abc
}

Reducing the iridium catalyst loading in the anode of polymer electrolyte membrane electrolyzers is a major goal to bring down the cost. However, anodes with low Ir-loading can suffer from poor electrical connectivity and hence lower the efficiency of the electrolyzer. In this work, we replace parts of the Nafion binder in the anode with an electrically conductive polymer (poly-3,4-ethylenedioxythiophene and polystyrene sulfonate acid complex, PEDOT:PSS) to counter this effect. At the optimal $50: 50$ blend we achieve a $120 \mathrm{mV}$ lower overpotential (2.02 V) at $3 \mathrm{~A} \mathrm{~cm}^{-2}$ compared to a pure Nafion reference $(2.14 \mathrm{~V})$. This corresponds to a $6 \%$ better efficiency. Ex situ resistivity measurements and high frequency resistance measurements indicate that the major cause for this improvement lies in the reduced electrical in-plane resistance due to the electrical conductivity of PEDOT:PSS.

Received 3rd August 2020

Accepted 5th October 2020

DOI: $10.1039 / \mathrm{dOra06714h}$

rsc.li/rsc-advances the CL results in a drop of the in-plane conductivity by two orders of magnitude, for ionomer contents higher than $3 \mathrm{wt} \%$. The authors suggest that the swelling of the ionomer causes a separation of the particles, resulting in a lower electron conductivity.

All of these approaches focused on improving performance by modifying either the catalyst material, the catalyst morphology or the electric contact to the catalyst material. In this work, we reduce the electrically insulating effect of the ionomer and thereby the in-plane resistivity in low-loaded anodic CLs by adding an electrically conductive polymer to the Nafion binder in the CL. We use poly-3,4ethylenedioxythiophene and polystyrene sulfonate acid complex (PEDOT:PSS), a commercially available conductive polymer. First developed as an antistatic material, ${ }^{8}$ PEDOT:PSS has become one of the most popular conductive polymers thanks to its high electrical conductivity, simple processability and high transmission of visible light. ${ }^{9}$ Due to these properties, it is most commonly used in organic photovoltaics, ${ }^{\mathbf{1 0 - 1 3}}$ OLEDs $^{14-16}$ and flexible electronics, ${ }^{17,18}$ and was studied in PEM fuel cells ${ }^{19-21}$ and supercapacitors. ${ }^{22-24}$ However, its application as conductive polymer binder in PEM water electrolysis has not yet been reported.

\section{Results}

\section{Electrochemical performance}

To investigate the impact of PEDOT:PSS in the anode binder on PEMWE performance, catalyst coated membranes (CCMs) with 
varying contents of PEDOT:PSS in the binder were fabricated on a N115 membrane (catalyst loading of $0.5 \mathrm{mg}_{\mathrm{Pt}} \mathrm{cm}^{-2}$ on cathode and $0.22 \mathrm{mg}_{\text {Ir }} \mathrm{cm}^{-2}$ on the anode side). The polarization curves of CCMs with 0 to $100 \%$ PEDOT:PSS are shown in Fig. 1a, where the total binder content was kept constant at $2 \mathrm{wt} \%$, while the ratio between Nafion and PEDOT:PSS was varied. It can be observed that the introduction of PEDOT:PSS in the anode CL has a strong impact on cell voltage, with a maximum reduced voltage of approximately $120 \mathrm{mV}$ at $3 \mathrm{~A} \mathrm{~cm}^{-2}$ when substituting $50 \%$ of the Nafion in the CL with PEDOT:PSS.

However, PEDOT:PSS causes an increase of the $i R$-free voltage as can be seen in Fig. 1b. Two effects can be observed that worsen the performance of the PEDOT:PSS samples. The Tafel slope (fitted between 10 and $100 \mathrm{~mA} \mathrm{~cm}{ }^{-2}$ ) is higher for the PEDOT:PSS samples (average of $56.7 \mathrm{mV} \mathrm{dec}^{-1}$ ) than for the reference (44.7 $\left.\mathrm{mV} \mathrm{dec}^{-1}\right)$, indicating worse kinetics. Furthermore, the sum of the mass transport and proton conduction losses can be estimated by the difference between the extrapolated Tafel fit and the high frequency resistance (HFR) free cell voltage as depicted by the grey area in Fig. $1 \mathrm{~b}$ (for a detailed explanation see ref. 25). With $83 \mathrm{mV} v s .61 \mathrm{mV}$ at $2 \mathrm{~A} \mathrm{~cm}^{-2}$, this sum is significantly higher for the PEDOT:PSS samples compared to the reference. Thus, PEDOT:PSS presumably
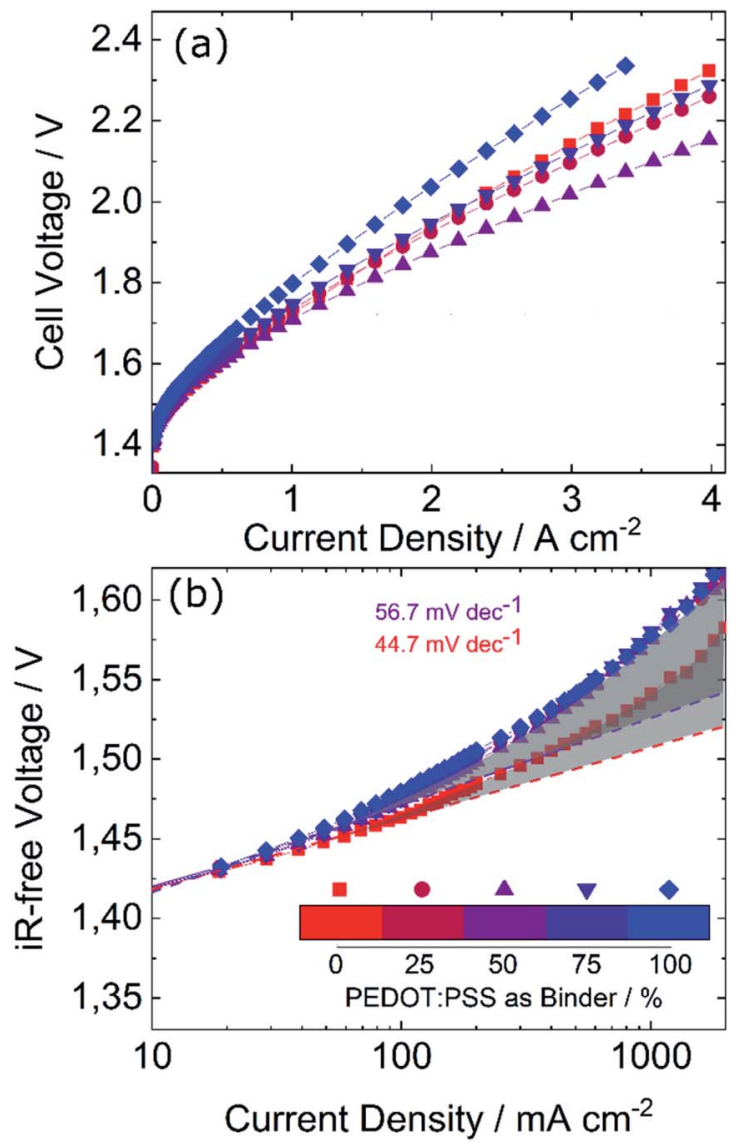

Fig. 1 Cell voltages (a) and $i R$-free voltage Tafel-plot (b) of MEAs with varying PEDOT:PSS contents, Nafion N115 membrane and 0.22 mg $\mathrm{cm}^{-2}$ loading at $80{ }^{\circ} \mathrm{C}$. A maximum reduction of the cell voltage was achieved for an equal binder content of PEDOT:PSS and Nafion. reduces protonic conductivity, which could be explained by the significantly lower protonic conductivity of PEDOT:PSS (1-13 $\mathrm{mS} \mathrm{cm}{ }^{-1}$ in $\left.1 \mathrm{M} \mathrm{H}_{2} \mathrm{SO}_{4}\right)^{26,27}$ compared to that of Nafion (80-100 $\left.\mathrm{mS} \mathrm{cm}^{-1}\right){ }^{28,29}$ However, as Tafel analysis does not allow to separate both loss mechanisms, mass transport resistance could also have a substantial contribution.

In spite of the increased $i R$-free voltage, PEDOT:PSS does reduce the overall cell voltage significantly as can be seen in Fig. 2a. The main reason for the reduced cell voltage therefore lies in a reduced high frequency resistance (HFR, Fig. 2b). The HFR corresponds to the sum of the protonic resistance in the membrane including the interface with the CL and the electric resistance in the CL including the interface with the Ti-PTL. ${ }^{30}$ Replacing Nafion with PEDOT:PSS should increase the electrical conductivity, which has a positive impact on the HFR. ${ }^{3}$ However, since the Nafion content is reduced at the same time, the protonic resistance increases with the PEDOT:PSS content. Fig. 2 shows also, that beyond 50\% PEDOT:PSS the HFR increases again. This could potentially stem from the reduced protonic conductivity at the interface with the membrane. To

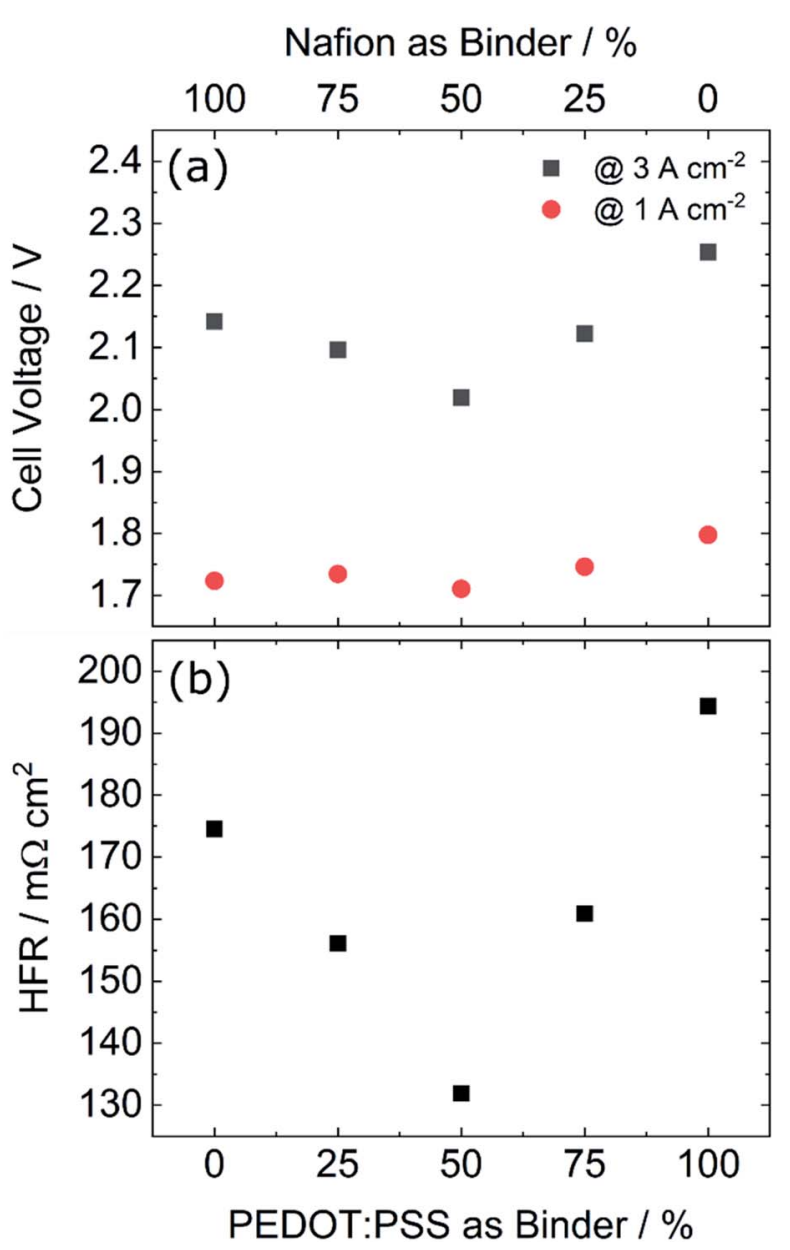

Fig. 2 Cell voltages of MEAs with varying PEDOT:PSS contents (a) and the corresponding HFR at $3 \mathrm{~A} \mathrm{~cm}^{-2}$. The total binder content was kept constant at $2 \mathrm{wt} \%$ relative to the catalyst mass. Both, the cell voltage and the HFR (b) show a minimum for a PEDOT:PSS content of $50 \%$ in the binder. 
further explore both hypotheses, an ex situ measurement of the electrical conductivity was conducted, presented in the next section.

\section{In-plane electrical conductivity}

In order to study the effect of PEDOT:PSS on the CL electrical resistivity, the sheet resistance of a series of test structures sprayed onto glass was measured, with a simple transfer line method. ${ }^{31,32}$ Two different loadings were measured: a lower loading of $0.22 \mathrm{mg}_{\mathrm{Ir}} \mathrm{cm}^{-2}$ (same as in the anodic CL) and a higher loading of $0.78 \mathrm{mg}_{\mathrm{Ir}} \mathrm{cm}^{-2}$, with increasing PEDOT:PSS contents.

The results are shown in Fig. 3a, where a linear reduction in the sheet resistance with increasing PEDOT:PSS content is observed. We also observe a stronger reduction in the sheet resistance (steeper slope) in the low loaded layers, compared to their higher loaded counterparts. This difference is most probably caused by the segmented nature observed in lower loaded layers, ${ }^{3}$ which results in isolated catalyst islands and

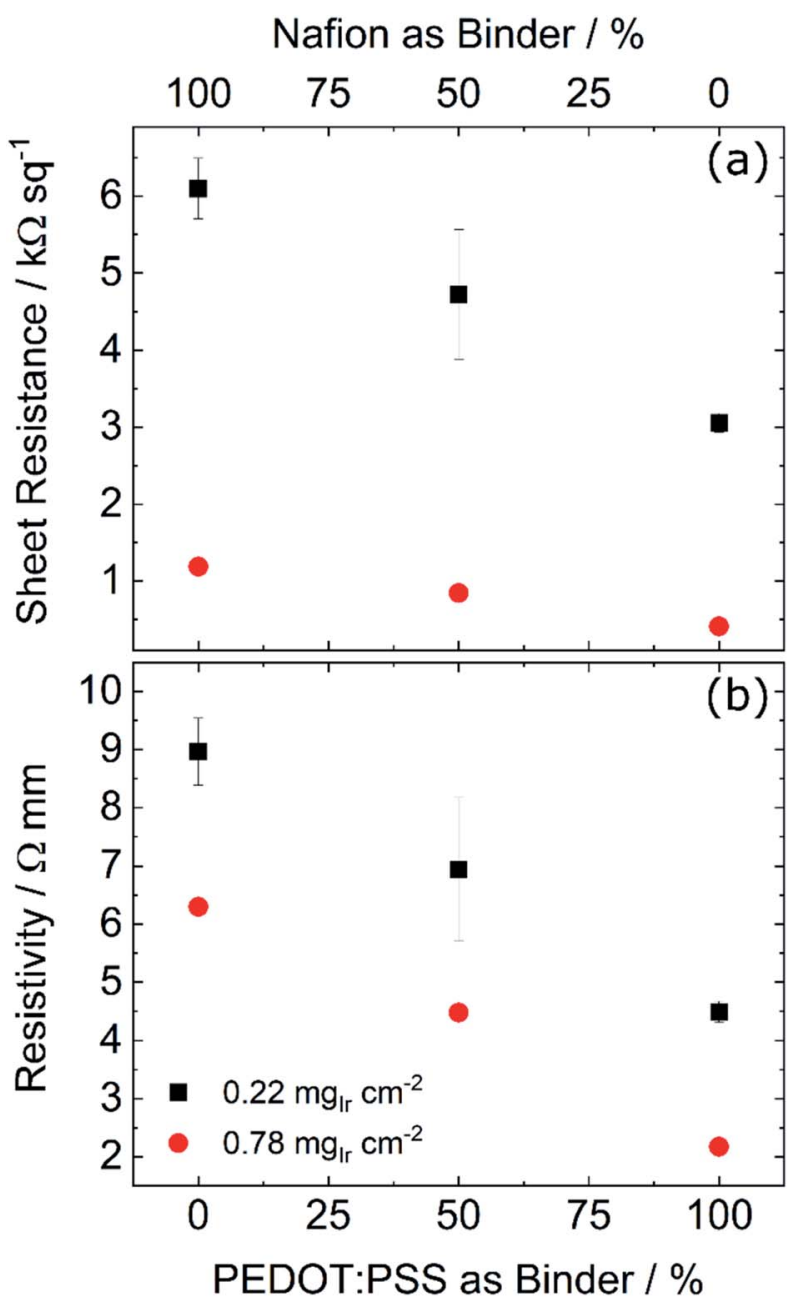

Fig. 3 Electronic sheet resistance of the catalyst layer determined ex situ (a) decreases with increasing PEDOT:PSS contents. The calculated resistivity (b) varied between high and low-loaded layers, likely caused by the presence of segmented sections in the $\mathrm{CL}$. longer pathways for the electrons. Moreover, the steeper reduction in the sheet resistance of the low-loaded layers indicates that the electron conduction in the low-loaded layers is stronger influenced by adding the conductive polymer as in the high loaded ones, where the electron pathways rely mostly on the catalyst. The higher resistivity of low-loading samples, as shown in Fig. 3b, further supports this hypothesis. Since both samples consist of the same material, the resistivity should be the same, regardless of thickness, for samples with the same PEDOT:PSS content. However, both curves have an offset of approximately $2.5 \Omega \mathrm{mm}$, which indicates that in this case the sample's resistivity increases as the thickness decreases. Although the test-structures were measured in dry conditions, the observed trends should hold true under different measuring conditions. Cross-sections of a low- and high-loading CL showing the difference in thickness are shown in Fig. S1. $\dagger$ These results show, that increasing the PEDOT:PSS content leads in fact to higher in-plane conductivities, which would explain the initial decrease in the HFR and the cell voltage up to the $50 \%$ minimum seen in Fig. 2b. The increase in HFR and cell voltage for higher PEDOT:PSS contents, could be explained by the reduced proton conductivity and possibly an increased interface resistance between the membrane and the CL due to the significantly lower proton conductivity of the PEDOT:PSS. Additionally, top view SEM images of the best performing and reference CLs showed no significant change in the layer's morphology, as shown in Fig. S2.† Potentially, these negative effects are initially compensated by the higher in-plane conductivity provided by the conductive polymer up to a certain point, after which the reduced proton conductivity and increased interface resistance cause the cell voltage and HFR to increase. Therefore the $50: 50$ ratio of Nafion and PEDOT:PSS represents the best compromise between electrical and protonic resistance.

\section{Conclusions}

A novel polymer blend of electrically and ionically conductive polymers (PEDOT:PSS and Nafion) was introduced as novel binder system for low-loaded Ir PEMWE anode catalyst layers in order to improve the electrical in-plane conductivity. An ex situ measurement of the catalyst layer's sheet resistance showed that increasing the conductive polymer content resulted in lower electrical resistances, with thinner layers presenting a higher reduction in sheet resistance, since resistivity increases with thinner layers due to their segmented nature. The reduced electric in-plane resistivity resulted in improved electrolysis performance: for a 50\% PEDOT:PSS blend, a reduction of $120 \mathrm{mV}$ at $3 \mathrm{~A} \mathrm{~cm}^{-2}$ in the cell voltage was obtained due to a lower high frequency resistance. PEDOT:PSS contents higher than $50 \%$ resulted in higher HFR values and cell voltages due to the increased protonic resistance and probably an increased interface resistance between the CL and the membrane.

The results presented in this work illustrate the relevance of improving the in-plane conductivity in low-loaded anodes. Compared to other approaches presented in recent studies, it is to note that the addition of PEDOT:PSS is a very simple and 
potentially cost-effective solution. PEDOT:PSS is a widely used low-cost polymer and blending with the ionomer allows straightforward integration into established MEA production processes and ink formulation. Furthermore, PEDOT:PSS could be easily combined with other strategies to improve the MEA's performance further. In this work no stability issues could be observed for the new polymer blend. However, future work should focus on the long-term stability of PEDOT:PSS in PEM water electrolyzers.

\section{Experimental}

\section{CCM fabrication}

All catalyst coated membranes (CCM) presented here were fabricated by applying catalyst ink on a membrane using an ultrasonic spray coater (Sonocell SNR-300) with the following parameters: frequency $130 \mathrm{kHz}$, spray head speed $170 \mathrm{~mm} \mathrm{~s}^{-1}$, pitch between spray paths of $1.5 \mathrm{~mm}$, ink flowrate of $0.35 \mathrm{ml} \mathrm{s}^{-1}$ and a hot plate temperature of $90^{\circ} \mathrm{C}$. All inks used to spray coat the anode consisted of $\mathrm{IrO}_{x}$ nano particles (Alfa Aesar, Premion, 99.99\%, Lot: W18E030) dispersed in a $1: 1$ Di water to 2-propanol solution. The total solids ratio in the suspension was $1 \mathrm{wt} \%$, the polymer content relative to the dispersed catalyst was kept constant at $2 \mathrm{wt} \%$ in all inks. Two different polymer dispersions were used: Nafion dispersion D520 from Fuel Cell Store and PEDOT:PSS $1.1 \mathrm{wt} \%$ dispersion in water, neutral $\mathrm{pH}$, high-conductivity grade, from Sigma Aldrich. The PEDOT:PSS content in the catalyst layer was varied in $0.5 \mathrm{wt} \%$ steps, starting from $0 \mathrm{wt} \%$ (i.e. $2 \mathrm{wt} \%$ Nafion) up to $2 \mathrm{wt} \%$ PEDOT:PSS. The total polymer content was kept constant for all samples to $2 \mathrm{wt} \%$, resulting in $0 \%, 25 \%, 50 \%, 75 \%$ and $1002 \%$ PEDOT:PSS content in the binder.

The cathode ink consisted of $60 \%$ Pt on high surface advanced carbon support (Hispec 9100, Lot: W12E007) dispersed in a $1: 4$ DI water to 2-propanol solution. The total solids ratio in the suspension was $2 \mathrm{wt} \%$ and the ionomer content (Nafion D2020) relative to the dispersed catalyst was $28 \mathrm{wt} \%$. All suspensions were prepared and ultrasonicated (Hielscher UIS250L) for $30 \mathrm{~min}$, with $100 \mathrm{~W}$, at a frequency of 24 $\mathrm{kHz}$ and in an ice bath before spraying.

\section{In-plane resistivity}

The in-plane resistivity was determined via transfer line method, using a similar method as Ahadi et al. ${ }^{32}$ Test structures were sprayed directly onto a glass substrate with a width of $1 \mathrm{~cm}$ and a length of $7.5 \mathrm{~cm}$. Four $0.5 \mathrm{~cm}$ wide carbon paper strips (Freudenberg H24C5) were simultaneously pressed against the test structure with a force of $3.7 \mathrm{~N}$. The resistance was measured at distances of $0.5,1.0$ and $1.5 \mathrm{~cm}$ in ambient conditions with a FLUKE 175 Multimeter. To calculate the in plane resistivity, the individual resistances were plotted against the corresponding distances and fitted linearly. We define the sheet resistance as follows: $R_{\text {sheet }}=\mathrm{d} R / \mathrm{d} x \times w$, where $\mathrm{d} R / \mathrm{d} x$ is the slope of the linear fit and $w$ the width of the test structure. From the sheet resistance, the in-plane resistivity was calculated as follows: $\rho_{\text {in-plane }}=R_{\text {sheet }} \times t^{-1}$, where $t$ is the thickness of the catalyst layer, obtained from cross sections of membranes coated alongside the test structure.

\section{Electrochemical performance}

All polarization curves presented here were measured using a Scribner 857 Redox Flow potentiostat with an active surface area of $5 \mathrm{~cm}^{2}$ in a single cell setup. For the anode side, a sintered titanium fiber PTL (Bekaert 2GDL40-1.0) was used and for the cathode side a PTL with MPL carbon paper from Freudenberg (H24C5). The cell was operated under ambient pressure, at $80{ }^{\circ} \mathrm{C}$ and with a flow of DI water of $40 \mathrm{ml} \mathrm{min}^{-1}$. Preheated DI water was pumped through the cell until the set value was reached, after which the voltage was held at $1.8 \mathrm{~V}$ until the current density stabilized. Polarization curves were recorded by holding the current constant for $120 \mathrm{~s}$ on each step. The high frequency resistance (HFR) was measured along the polarization curve with a frequency of $1 \mathrm{kHz}$.

\section{Conflicts of interest}

There are no conflicts to declare.

\section{Acknowledgements}

The authors gratefully acknowledge financial support of this work by the Federal Ministry of Education and Research in Germany within the project NeutroSense (grant number: 05KI9VFA) and by the BMBF within the project "ForLab" (grant number: 16ES0943).

\section{References}

1 M. Carmo, D. L. Fritz, J. Mergel and D. Stolten, Int. J. Hydrogen Energy, 2013, 38(12), 4901.

2 P. T. Moseley and J. Garche, Electrochemical Energy Storage for Renewable Sources and Grid Balancing, Elsevier Science, 2014.

3 M. Bernt, A. Siebel and H. A. Gasteiger, J. Electrochem. Soc., 2018, 165(5), F305-F314.

4 C. Rozain, E. Mayousse, N. Guillet and P. Millet, Appl. Catal., $B, 2016,182,123$.

5 M. Yasutake, H. Anai, D. Kawachino, Z. Noda, J. Matsuda, K. Ito, A. Hayashi and K. Sasaki, ECS Trans., 2018, 86(13), 673.

6 T. Schuler, J. M. Ciccone, B. Krentscher, F. Marone, C. Peter, T. J. Schmidt and F. N. Büchi, Adv. Energy Mater., 2019, 10(2), 1903216.

7 F. Hegge, F. Lombeck, E. Cruz Ortiz, L. Bohn, M. von Holst, M. Kroschel, J. Hübner, M. Breitwieser, P. Strasser and S. Vierrath, ACS Appl. Energy Mater., 2020, 8276-8284.

8 S. Kirchmeyer and K. Reuter, J. Mater. Chem., 2005, 15(21), 2077.

9 Y. Wen and J. Xu, J. Polym. Sci., Part A: Polym. Chem., 2017, 55(7), 1121.

10 K. Rohtlaid, G. T. M. Nguyen, C. Soyer, E. Cattan, F. Vidal and C. Plesse, Adv. Electron. Mater., 2019, 5(4), 1800948. 
11 K. Sun, S. Zhang, P. Li, Y. Xia, X. Zhang, D. Du, F. H. Isikgor and J. Ouyang, J. Mater. Sci.: Mater. Electron., 2015, 26(7), 4438.

12 Z. Zhao, Q. Liu, W. Zhang and S. Yang, Sci. China: Chem., 2018, 61(9), 1179.

13 K. Rohtlaid, G. T. M. Nguyen, C. Soyer, E. Cattan, F. Vidal and C. Plesse, Adv. Electron. Mater., 2019, 5(4), 1800948.

14 Z.-Z. Gu, Y. Tian, H.-Z. Geng, D. S. Rhen, A. S. Ethiraj, X. Zhang, L.-C. Jing, T. Wang, Z.-H. Xu and X.-T. Yuan, Appl. Nanosci., 2019, 9(8), 1971.

15 D. Hewidy, A.-S. Gadallah and G. A. Fattah, J. Mol. Struct., 2017, 1130, 327.

16 Q. Zheng, F. You, J. Xu, J. Xiong, X. Xue, P. Cai, X. Zhang, H. Wang, B. Wei and L. Wang, Org. Electron., 2017, 46, 7.

17 J. Chang, J. Shang, Y. Sun, L. K. Ono, D. Wang, Z. Ma, Q. Huang, D. Chen, G. Liu, Y. Cui, Y. Qi and Z. Zheng, Nat. Commun., 2018, 9(1), 4480.

18 Y. Yang, H. Deng and Q. Fu, Mater. Chem. Front., 2020, DOI: 10.1039/D0QM00308E.

19 K. K. Tintula, S. Pitchumani, P. Sridhar and A. K. Shukla, Bull. Mater. Sci., 2010, 33(2), 157.

20 H. Kim, Y.-J. Lee, G.-G. Park, S.-H. Park, Y.-Y. Choi and Y. Yoo, Carbon, 2015, 85, 422.

21 E. Daş and A. Bayrakçeken Yurtcan, Energy Technol., 2017, 5(9), 1552.
22 P. G. Pickup, C. L. Kean, M. C. Lefebvre, G. Li, Z. Qi and J. Shan, J. New Mater. Electrochem. Syst., 2000, 3, 21.

23 G. Cai, P. Darmawan, M. Cui, J. Wang, J. Chen, S. Magdassi and P. S. Lee, Adv. Energy Mater., 2016, 6(4), 1501882.

24 C. Zhang, T. M. Higgins, S.-H. Park, S. E. O'Brien, D. Long, J. N. Coleman and V. Nicolosi, Nano Energy, 2016, 28, 495.

25 M. Suermann, K. Takanohash, A. Lamibrac, T. J. Schmidt and F. N. Büchi, J. Electrochem. Soc., 2017, 164, F973-F980.

26 M. C. Lefebvre, Z. Qi and P. G. Pickup, J. Electrochem. Soc., 1999, 2054.

27 G. Li and P. G. Pickup, Phys. Chem. Chem. Phys., 2000, 2(6), 1255.

28 M. Yoshitake, M. Tamura, N. Yoshida and T. Ishisaki, Denki Kagaku, 1996, 64, 727.

29 N. Yoshida, T. Ishisaki, A. Watakabe and M. Yoshitake, Electrochim. Acta, 1998, 43(24), 3749.

30 D. G. Bessarabov, H. Wang, H. Li and N. Zhao, PEM electrolysis for hydrogen production: Principles and applications, CRC Press, Boca Raton, Florida, 2016.

31 G. K. Reeves and H. B. Harrison, IEEE Electron Device Lett., 1982, 3(5), 111.

32 M. Ahadi, M. Tam, J. Stumper and M. Bahrami, Int. J. Hydrogen Energy, 2019, 44(7), 3603. 